\title{
It's all about the feels: How and why students' feelings about their engineering program change
}

\section{Dr. Laura Hirshfield, University of Michigan}

Laura Hirshfield is a lecturer and researcher at the University of Michigan. She received her B.S. from the University of Michigan and her Ph.D. from Purdue University, both in chemical engineering. She then transitioned into the engineering education field with postdoctoral positions at Oregon State University, Olin College of Engineering and University of Michigan. Her research interests lie in assessing and amending curricula to help students transition from undergraduate to professional practice.

\section{Stacie Edington, University of Michigan}

Stacie Edington is the Director of Honors and Engagement Programs within the University of Michigan, College of Engineering. She received her Bachelor of Arts in Sociology from the University of Michigan and her Master of Science in Executive Leadership from the University of San Diego.

\section{Michael Dailey, University of Michigan}

Mike serves as the Assistant Director for the Engineering Honors and Engagement Programs Office, which houses the Common Reading Experience for the College of Engineering at the University of Michigan.

Mike earned his BBA in Management from Eastern Michigan University. He also earned a Graduate Certificate in Academic Advising and Masters of Arts in Higher Education Administration from Sam Houston State University. 


\section{Introduction}

How students feel about starting and being an engineering student will assuredly influence and, subsequently, be impacted by students' college experiences. A student who feels more positively about their experience in college (or in general) will probably be more likely to rate highly on many other aspects related to affect, such as confidence, self-efficacy, engagement, or persistence. Similarly, a student who feels negatively may be less likely to find community, succeed in their coursework, or to persist in the engineering major. There are many tools related to gauging elements of students' affect that can be immensely useful in encouraging students and helping them succeed. However, simply gauging students' feelings may also give insight into their experiences as an engineering student.

In this work, we focus on data that resulted from a larger study investigating students' perceptions of engineering, sense of belonging, and sense of community as they participate in a Common Read first-year program. In the study, incoming first-year students participated in a pre- and mid-summer survey and a post-program survey. A subset of these students also participated in focus groups, held with students of all levels who were prompted to reflect on their experiences starting college and participating in first-year programs. This study focuses on data gathered in the mid-summer survey and the focus groups, specifically, that gauged how students felt starting engineering college.

The findings presented here aim to investigate how students feel as incoming first-year engineering students, and what elements of their experience may relate to those feelings. If we can better understand how students feel, we can better support and encourage them, and help them to develop more positive affect and to succeed more easily. The work presented here is guided by the following research questions:

1. How do incoming first-year students feel about starting engineering college?

2. How do engineering students describe their feelings about being accepted to and starting engineering college?

3. What aspects of the student experience do students identify as causing or relating to those feelings?

\section{Background}

\section{Feelings and Affect}

The term affect can refer to several aspects of a student experience that relate to feeling or emotion, as opposed to cognition or behavior. A student's affect has the capability to greatly impact their school experience: it has also been found that a positive affect correlates to higher success in school [1]. A student's emotions can impact their cognitive functioning [2], their emotional intelligence and abilities to work with other students [3] and can affect ethical decision making [4]. However, beyond considering how affect impacts other elements of a student experience, it is also important to directly gauge the status of a student's affect and how it develops to ensure that a student has positive experiences and positive emotions during their college experience. 
Affect is usually a term used broadly to describe many elements that relate to a student's emotional state. For example, one element of affect that is commonly studied in engineering education is confidence, or how a student develops self-esteem, and self-efficacy, or belief in oneself to be able to succeed [5], [6]. Sense of community [7], [8] or sense of belonging [9], [10] are two other elements of affect, describing how a student finds their place in a group and feels that they relate to others. Motivation, engagement, and persistence also relate to affect, considering how a student interacts with their work and finds the desire to succeed or persevere.

However, in this work, the word "feelings" is used as a more colloquial reference to a student's affect more generally, in which affect is considered to describe a student's emotions, feelings, or mood [11]. Although the previously-mentioned elements of affect are more commonly studied in educational settings, there have been more recent studies focusing more pointedly on students' feelings as part of their experience [12], such as how students' feelings change throughout an integrated learning experience [13] or throughout a leadership training [14]. Studies focused on adolescents specifically have found that a student's feelings can greatly impact their academic experience: students with positive feelings will have better cognitive capabilities, more adaptability, more active participation, and form more healthy relationships [15], [16] whereas students with negative feelings will have less motivation and drive to succeed [17], [18]. In this work, the authors were inspired to investigate how students feelings change after starting college, as it has been found that students' emotional health declines while starting at university and the decline is even more severe for female students [19].

As it is used here, the word "feelings" encompasses all of the previously-mentioned elements of affect (confidence, sense of belonging, etc.); but also, a student's "feelings" can affect and be impacted by each of the different elements of affect. For example, feelings can often be considered to be measured by a students' physiological state [20]; and one contributor to selfefficacy (an aspect of a student's affect) is physiological state [5]. If a student has an upset stomach or dizziness - in other words, symptoms of anxiety - they may experience reduced selfefficacy. Whereas if they experience an elevated heart rate or increased blood rush to the head, symptoms that can be associated with being excited, they may experience an increase in selfefficacy. In other words, a student's most basic feelings will both be influenced by and, in turn, influence, their self-efficacy.

Therefore, while it is recognized that it is important to study how different elements of student's affect are developed or impacted by their college experience, we may also consider a most basic form of a student's affect - their feelings - to assess a student's perceptions of their experience. Describing feelings can be a more accessible way for students to report, gauge, or reflect on their own affect, as people are taught from a young age to describe their emotions in simple terms (happy, sad, angry). Thus, here, we consider how students describe their feelings about being accepted to and starting college, as a proxy for considering their affect overall.

\section{Impact of Identity on Feelings}

Another element of affect is identity, or how a student views themselves [21]; if a student does not view themselves as part of a field or community, they are unlikely to "feel" as if they are 
going to succeed [22]. Relatedly, a student's identity can have great impact on the emotions that they have, interpret, or express, and it is important to note that a student's identity has great impact on the way they participated and engaged throughout the data collection presented in this paper.

For example, where a student is from can impact the emotions that they feel comfortable expressing. In a study focused on students from Germany, Israel, Greece and the United States, researchers found differences in how students from different countries perceive emotion, in particular, anger and sadness [23]. Certain emotions are valued differently in Western cultures, or individualist cultures, compared to Eastern, or collectivist, cultures [24]. Western cultures tend to put more emphasis on "high arousal" feelings - excited, frustrated, happy, enthusiastic, nervous - whereas Eastern cultures emphasize "low arousal" feelings, like calm, contended, pleased, satisfied, depressed, relaxed [25]-[28]. Thus, students who were raised in a Western culture will be more comfortable expressing and more likely to identify feeling these "high arousal" emotions, whereas students from an Eastern culture will more likely to use "low arousal" feelings to describe their emotional state.

In considering gender identity, there is a stereotype that women experience and express a wider range of emotions than men do, except for men more often express anger and pride [29]; students who are aware of those stereotypes may be inclined to adhere to them, and this can be particularly damaging for male students who feel that they cannot express their full range of emotions. "Masculinity ideology" is a set of beliefs about the importance of men adhering to culturally-defined standards of male behavior [30]; as it pertains to feelings, this can explain how men will be more likely to not interpret or express the wide range of emotions that women may feel more comfortable with. For example, instead of expressing or properly interpreting more stereotypically female emotions, such as sadness or vulnerability, men may find it more socially acceptable to express those emotions as anger [31].

Race and ethnicity can also impact a student's expression of feelings. For example, the racism that African American people encounter may lead to a lowered sense of self-importance which can affect their willingness to consider their feelings as valid [32]. The concept of masculinity ideology, discussed previously, can be particularly prevalent for men of color [33], as they are further marginalized and may not find it as easy to break out of traditional, stereotypical gender roles [34].

\section{Methods}

The study presented here is part of a larger study investigating the impact of a first-year Common Read Experience program on students' sense of community, sense of belonging, and perceptions of engineering [35][36]. The focus of the work presented here is specifically on students' discussions of feelings and emotions about starting and being in an undergraduate engineering program, and how their feelings (or perceptions of their feelings) changed throughout their time in the program.

\section{Data Collection}


In the study, all first-year students starting in an engineering program at a large, Midwestern university were invited to complete surveys at the beginning of the summer $(n=731)$ and during the fall semester $(n=1128)$ and responded to questions related to sense of belonging, community, and perceptions of engineering. However, in this paper, the focus will be specifically on data collected in a mid-summer survey and focus groups held during the fall semester.

In the middle of summer, students participated in a third survey (called the mid-summer survey, $\mathrm{n}=1171$ ) that included a question that aimed to generally gauge students' feelings about their impending start in the engineering college. The question asked students to fill in the blank in the sentence "I am __ to be an engineer [at this university]." 812 students (approximately $70 \%$ of the mid-summer survey respondents) responded to this specific question. Demographic data was not collected in the mid-summer survey.

A subset of students also participated in focus groups during the fall semester that focused on discussions around students' feelings about college, their senses of belonging and community, and their participation in the Common Read Experience program. While discussing these topics, students typically reflected on how it felt to start in the engineering college as a first-year student; these reflections provided the majority of the findings related to feelings that are presented in this paper. While these focus groups concentrated on first-year experiences, separate groups were held with students at all levels to aim to gauge how their perceptions of starting college and first-year programs changed as they continued on in their college program. A total of sixty students participated in the focus groups, ranging from first-year students to senior students.

\section{Data Analysis}

The data resulting from the mid-summer survey was iteratively and emergently coded in order to identify common trends among student answers [37]. First, the responses were grouped and quantified (i.e. all of the students who wrote "excited" were collapsed into one response, and assigned an "N" of 327 since 327 students wrote that word). Next, one of the authors of the paper coded the responses to have "positive," "neutral" or "negative" connotations (or, in some cases, words were coded to be both positive and negative). Next, within those categories, words were grouped together with a common sentiment. In some cases, the groupings were clear; for example, "excited" and "very excited" both relay an "excited" sentiment. For other words, a thesaurus was used to determine word relation. In some cases, responses belonged to multiple sentiments (for example, "thrilled and proud" denotes both an "excited" sentiment and a "proud" sentiment) and in the case that they were counted in multiple categories. After all of the responses had been coded, the other two authors of the paper read over all of the codes and noted disagreement. These disagreements were then discussed between all three authors, and a compromise was reached for the final set of codes.

The results from the mid-summer survey data were used as a framework for analysis of the focus group findings. The focus group recordings were first transcribed, then coded with a codebook encompassing each of the identified sentiments from the mid-summer survey. Student responses were coded according to directly saying they felt a certain sentiment, or by conveying a certain sentiment (i.e. "excited" or "happy"). These coded excerpts were then considered in relation to 
the mid-summer survey findings, in an effort to contextualize students' feelings about starting and attending engineering college.

\section{Results}

\section{Mid-Summer Survey Findings}

When students filled in the blank in "I am __ to be an engineer [at this university]," the majority of responses (662, or 82\%) were coded as positive words or phrases (Table 1). The most common sentiment was "excited" with 481 (59\%) responses denoting some sort of excitement about attending engineering college, with "excited" specifically being the most common response, from 327 (40\%) of students. The next most common sentiment was "proud," with 124 $(15 \%)$ of students expressing some sort of pride about attending the college of engineering. Other positive sentiments were "fortunate," "happy," "challenged," and "motivated."

Table 1. Positive responses to "I am to be an engineer [at this university]."

\begin{tabular}{|c|c|c|}
\hline Response & Sentiment & $\mathbf{N}$ \\
\hline Excited & \multirow[t]{23}{*}{ Excited } & 327 \\
\hline Thrilled & & 36 \\
\hline Ecstatic & & 33 \\
\hline Eager & & 15 \\
\hline Pumped & & 7 \\
\hline Stoked & & 5 \\
\hline Elated & & 4 \\
\hline Extremely excited & & 4 \\
\hline Hyped & & 4 \\
\hline Proud and excited & & 4 \\
\hline So excited & & 4 \\
\hline Very excited & & 4 \\
\hline Beyond excited & & 3 \\
\hline Enthusiastic & & 3 \\
\hline Looking forward & & 3 \\
\hline Excited and enthusiastic & & 2 \\
\hline Primed and excited & & 2 \\
\hline Ready and excited & & 2 \\
\hline Absolutely stoked & & 1 \\
\hline $\begin{array}{l}\text { Absolutely, positively, ready-to-take-on- } \\
\text { college and beyond thrilled :D }\end{array}$ & & 1 \\
\hline Amazed & & 1 \\
\hline Amped & & 1 \\
\hline Electrified & & 1 \\
\hline
\end{tabular}




\begin{tabular}{|c|c|c|}
\hline Enthralled & & 1 \\
\hline Excited and proud & & 1 \\
\hline Exhilarated & & 1 \\
\hline Frickin' pumped & & 1 \\
\hline Generally excited & & 1 \\
\hline More excited than a kid in a candy shop & & 1 \\
\hline Psyched & & 1 \\
\hline Really excited & & 1 \\
\hline Thankful, proud, and excited & & 1 \\
\hline Thrilled and proud & & 1 \\
\hline Thrilled, honored, and prepared & & 1 \\
\hline Unequivocally excited & & 1 \\
\hline Very enthusiastic & & 1 \\
\hline Zealous & & 1 \\
\hline Proud & Proud & 114 \\
\hline Proud and excited & & 4 \\
\hline Darn proud & & 1 \\
\hline Excited and proud & & 1 \\
\hline Proud? Is this a trick question? & & 1 \\
\hline Thankful, proud, and excited & & 1 \\
\hline Thrilled and proud & & 1 \\
\hline Thrilled, honored, and prepared & & 1 \\
\hline Honored & Fortunate & 20 \\
\hline Grateful & & 3 \\
\hline Blessed & & 2 \\
\hline Lucky & & 2 \\
\hline Extremely grateful & & 1 \\
\hline Grateful and excited & & 1 \\
\hline Humbled & & 1 \\
\hline Fortunate & & 1 \\
\hline Privileged & & 1 \\
\hline Happy & Happy & 11 \\
\hline Glad & & 5 \\
\hline Delighted & & 1 \\
\hline Jubilant & & 1 \\
\hline Pleased & & 1 \\
\hline Inspired and challenged & Challenged & 2 \\
\hline Up to the challenge & & 1 \\
\hline Motivated & Motivated & 3 \\
\hline
\end{tabular}


118 students (15\%) included words or phrases that were coded as neutral sentiments (Table 2). The most common neutral sentiment was "ready," (80 students, or 10\%) implying that they were going to start college soon, albeit there were varying degrees of certainty (ranging from "most likely going" to "definitely going"). Some sentiments were more action-based and had to do with aiming, in various degrees, towards being an engineer, like "determined" or "hoping."

Table 2. Neutral responses to "I am

\section{Response}

Going

Ready

About

Planning

Already

Bound

Certain

Definitely going

Most likely going

Ready to learn how

Determined

Striving

Aiming

Committed

Convinced

Dying

Going to work my hardest

Going to fight to the bitter end

Gonna go ham and study a helluva lot

Hungry

Willing to work hard in order

Hoping

Aspiring

Yearning

Born

Called

Destined

Meant

Intrigued

Curious

Flabbergasted to be an engineer [at this university]." Sentiment N Ready 50

19

3

3

1

1

1

1

1

Determined 8

\section{-}

3

3

1

$\frac{1}{1}$

1

1

- $\frac{1}{1}$

\begin{tabular}{lr} 
Hoping & $\frac{3}{2}$ \\
\cline { 2 - 2 } Destined & $\frac{1}{1}$ \\
\hline & $\frac{1}{1}$ \\
\hline Curious & $\frac{1}{1}$ \\
\hline Surprised & 2 \\
\hline
\end{tabular}




\begin{tabular}{lll}
\hline Challenged & Challenged & 1 \\
\hline Paying & Paying & 1 \\
\hline
\end{tabular}

17 students (2\%) included responses that were coded as negative sentiments (Table 3 ). The most common negative sentiment (13 students, or $2 \%$ ) had to do with being "stressed" associated with becoming an engineering student. Two students implied they were intending to transfer out of the engineering college to a different major.

Table 3. Negative responses to "I am to be an engineer [at this university]."

\begin{tabular}{|c|c|c|}
\hline Response & Code & $\mathbf{N}$ \\
\hline Anxious & \multirow[t]{5}{*}{ Stressed } & 5 \\
\hline Nervous & & 5 \\
\hline Hoping I am good enough & & 1 \\
\hline Mentally preparing myself for all of the stress that comes with wanting & & 1 \\
\hline Worried & & 1 \\
\hline Not going (switching colleges) & \multirow[t]{2}{*}{ Not } & 1 \\
\hline Not planning & & 1 \\
\hline Meh; slightly looking forward to yet at the same time slightly disappointed & Disappointed & 1 \\
\hline Uninspired & Uninspired & 1 \\
\hline
\end{tabular}

There were also seven responses (1\%) that had both positive and negative connotations, all having to do with feeling both excited and nervous (Table 4).

Table 4. Positive/negative responses to "I am Response to be an engineer [at this university]."

Excited and nervous $\mathbf{N}$

Extremely excited, a little bit nervous but really happy to have an opportunity

Slightly nervous but very excited

The remainder of the responses (eight, or 1\%) were answers unrelated to feelings, such as "supercalifragilisticexpialidocious" or "18 years old."

\section{Focus Group Findings}

Although $81 \%$ of students had positive feelings about starting engineering college, according to the mid-summer survey, positive sentiments were rarely expressed in the focus groups with regards to starting their first year of engineering college. Being "excited," in particular, was the single most common response on the survey, but was only discussed a few times, and always by women. One woman said:

I didn't know how I'd feel until I actually got accepted to [this school] and I was just like really excited. That was the only [acceptance] that made an impact on 
me. And so I was like, I feel like I should go here because this is the only one that actually I was actually excited about, like knowing that I could go here.

Another woman directly related her excitement to being a woman in engineering:

I think it was kind of validating for me as a woman in engineering that I could kind of cut it. I mean I'm sure there's people that would tell me later on that I only got in because I'm a woman, but at the time it felt nice. I was really excited.

$14 \%$ of students reported "neutral" sentiments on the survey, but more students discussed their feelings about starting college in the focus group expressed neutral sentiments than positive sentiments. One underrepresented minority male student expressed the sentiment of being determined to succeed in college:

I think part of the reason that I came here to what is like the doubt associated with me and not getting in. So there's a lot of people, even today, where they can't see me getting into [this school], totally. Like "you can't go there. You can't have gotten into [that school], one of the top public schools." So that was when I did get accepted, that was part of the reason why I was like ... I'll show you.

Another neutral sentiment expressed commonly in the focus groups was having to do with money. Although only one student had a response related to finances on the survey (I am "paying" to be an engineer [at this university]), many students discussed coming to this engineering college for a reason related to finances. Most of those students expressed wanting to come to this college due to receiving financial aid, scholarships, or choosing this university as opposed to out-of-state schools due to the lower cost of in-state tuition. However, one student discussed being accepted to the university in both a positive and negative way related to finances; interestingly, the positive and negative sentiments expressed that paralleled the only "positive and negative" sentiment from the survey: excited and nervous. Also, in accordance with all positive sentiments being expressed by female or underrepresented students, this "positive and negative" response was discussed by an underrepresented male student. The student was both excited to come to school, but dismayed about how much it would cost as an out-of-state student:

I was excited, but then I was immediately dismayed because of the tuition, being out-of-state. [My dad] was looking at this huge $\$ 50,000, \$ 60,000$ number, and I was wondering how am I going to even pay for this? ... when [my dad] told me [that I could use the Montgomery GI Bill to help pay for college] I was extremely excited too because I had a way to get in or a way to actually pay for school. 'Cause Lord knows I couldn't afford it.

Another commonly-expressed neutral or negative sentiment seemed to mirror the person who responded to the survey with the negative sentiment of "I am meh; slightly looking forward to yet at the same time slightly disappointed to be an engineer [at this university]." Several students discussed being unsurprised about their acceptance, and although they did not express disappointment, they were not necessarily excited to attend the university. For example: 
I felt ... this is cynical, I don't know, I was just like, "oh, okay, cool." I wasn't surprised, honestly. I told my mom and she was like, [excitedly] "oh, [she] just got accepted at [this school]! Did you get your letter?" Yeah, mom. I got the email three hours ago.

Finally, only $2 \%$ of survey responses were coded as negative, yet that was the most common sentiment expressed in the focus groups when students discussed being accepted to or starting engineering college. All but two of the students with negative sentiments were male students who were not underrepresented minorities. One of the most commonly expressed negative sentiments was "overwhelmed," despite this not being a response on the survey at all. One male student said: "I definitely felt that sense of ... coming in [to college], it's a lot." One female student said:

I felt like freshman year, I didn't really feel like I was connected to the engineering community just cause like you're taking all these pre-req classes but for [one of the two introductory engineering classes] I felt like was just like overwhelming. There were so many people in that first class and the office hours were horrible. Like just, I absolutely hated that class... It's just like when you needed help you were on your own and that was really tough to go through for your very first semester.

\section{Discussion}

In comparing the two datasets, the focus group responses seemed to contradict the findings from the mid-summer survey. Most students used negative sentiments when they reflected on being accepted to or starting engineering college, with many students expressing feeling overwhelmed, although no students using a word related to that on the survey. This finding aligns with another study finding that students' emotional health declines over the beginning of their college career [19]. Many students also expressed a somewhat neutral or negative sentiment about being accepted to the engineering college, noting that it was something that was expected of them or was simply not exciting to them. Several students discussed finances when discussing their feelings about starting school, even though only one student used a word related to finances on the survey. Most students discussed finances in a positive way (in that they received financial aid or a lower tuition at this university) but one student discussed it as being a detriment to their excitement about starting college.

The fact that the focus group findings were seemingly contradictory to the survey findings could be attributed to many factors. One obvious reason could be that students' feelings change drastically once they actually start school; in the survey, they haven't yet started college and may not be feeling many pressures associated with that transition, while in the focus groups, they have already been in college. Another reason could be attributed to students' responding about their feelings in the moment (when responding to the survey) versus reflecting on them; it appeared that students imposed a more neutral or negative sentiment when thinking about how they felt, whether that was three months or three years later than when they were first accepted. Their feelings could be colored by their experiences they had since being incoming first-year 
students, or students could be experiencing a negativity bias where they recall their more negative emotions more vividly than the positive ones. Similarly, students could be responding differently about their feelings because of the context; students typing out their feelings, alone, in a relatively quick survey could lead to different reports than when students are verbally processing their feelings in a focus group among others.

Another interesting finding from the focus groups, specifically, was that the responses seemed split down gender and race/ethnicity lines; underrepresented students (women and underrepresented minorities) were the only ones to report positive sentiments about being accepted to or starting engineering college, reflecting on being excited or happy. Also, the majority of negative responses were from non-underrepresented students (Caucasian or Asian/Asian-American men), discussing feeling overwhelmed about starting school.

It is important to note that this study has limitations. First, this data is focused on only sixty students attending one university, and so the findings cannot necessarily be transferable to a different set of students at a different institution. The coding done of the survey data is limited by lack of interaction with the participant; the authors had to make assumptions as to the intended meaning of the survey respondents while categorizing feelings as "positive," "negative," or "neutral" and could not take nuance or context into account. Also, the focus group results are limited because the data is largely retrospective, and students are considering their feelings from one to three years prior, as noted previously. There is also a limitation associated with making comparisons between the findings from the two different datasets we considered for this paper; we are comparing results from two sets of students that may not overlap, and we are comparing findings gathered in two very different contexts: one context where a student is responding individually to a survey in the moment, and second where students are surrounded by peers, verbally reporting their feelings retrospectively. Because of these limitations, we cannot assert with certainty that students' feelings $d o$ change over the course of their college experience according to our results, but we can note that they do report perceiving their feelings differently at different points in time.

\section{Conclusion}

The work presented here, part of a larger study focusing on sense of community and belonging for first-year students, is aimed at investigating how students describe their feelings about starting engineering college. Students' feelings were assessed in a mid-summer survey where students filled in the blank on "I am __ to be an engineer [at this university]." In the midsummer survey, the majority of incoming first-year students were positive about being an engineer, with $81 \%$ of students using positive sentiments like excited, proud, fortunate, happy, challenged, and motivated to fill in the blank. $59 \%$ of students used a sentiment related to excitement, with the single most popular answer being "excited," coming from $40 \%$ of students. $15 \%$ of students used neutral sentiments, that could be perceived either positively or negatively depending on context, or were simply stating fact. Neutral sentiments included ready, determined, hoping, destined, curious, surprised, challenged, or paying. Only $2 \%$ of students used negative sentiments, such as stressed, not (meaning they were or already had dropped the major), disappointed, or uninspired. In focus groups held after students started college, however, students reflected on how they felt upon starting school, and students were generally more likely 
to report negative or neutral sentiments, although underrepresented students (women and underrepresented minorities) still reported feeling excited or happy upon starting college.

These findings illuminate that, particularly before starting college, students are mostly positive about starting their engineering studies, and instructors should do their best to capitalize on these positive attitudes as they deal with the challenges of starting engineering college. Instructors could engage in efforts to harness that excitement to deepen interest and joy of learning in engineering. As students begin their journey in engineering, they generally start with many large lecture classes and may quickly lose interest in the subject if not engaged to cultivate their excitement. Instructors should also consider that students' feelings about starting college may be different based on their identity, and even if students are feeling positively, it does not mean that they are having a seamless experience; or, similarly, negative emotions may not be indicative of a very difficult time. Still, in the absence of being able to formally assess other metrics related to student affect (like confidence, self-efficacy, motivation, engagement, etc.), simply gauging how students feel may be sufficient to know how to best support students as they begin the path to become engineers.

The findings discussed here illuminate several potential directions for future work. In future studies, it is important to investigate the discrepancy between the mid-summer survey and the focus groups and what contributing factors might exist. Conducting additional research using the same survey techniques at both periods of time would be beneficial. Students may have forgotten how they felt before coming to the university since they were answering focus group sessions as current students. Because the results were so dramatically different, doing another survey after school begins with the same fill-in-the-blank prompt may show progress from the excitement of summer to the negative sentiments received once school began. It would also be important to understand differences in the survey responses based on demographics. Future work should include an exploration of why women and underrepresented minority students provided such positive experiences as current students in the focus group compared to Caucasian and Asian/Asian-American male students. An exploration of social identity, including intersectionality, and the related differences in expression of emotion could be beneficial. Additionally, future work could include a longitudinal study of students' feelings prior to entry through graduation, in comparison with academic success and perseverance toward degree.

\section{References}

[1] M. Weber, L. Wagner, and W. Ruch, "Positive feelings at school: On the relationships between students' character strengths, school-related affect, and school functioning," $J$. Happiness Stud., vol. 17, no. 1, pp. 341-355, 2016.

[2] C. M. Tyng, H. U. Amin, M. N. M. Saad, and A. S. Malik, "The influences of emotion on learning and memory," Front. Psychol., vol. 8, no. 1454, 2017.

[3] M. J. Riemer, "Integrating emotional intelligence into engineering education," World Trans. Eng. Technol. Educ., vol. 2, no. 2, pp. 189-194, 2003.

[4] D. Kim and B. K. Jesiek, "Work-in-Progress: Emotion and intuition in engineering students' ethical decision-making and implications for engineering ethics education," 2019.

[5] A. Bandura, Self-Efficacy: The Exercise of Control. New York, NY: Freeman, 1997. 
[6] F. Pajares, "Self-efficacy in academic settings," in American Educational Research Association, 1995.

[7] D. W. McMillan and D. M. Chavis, "Sense of community: A definition and theory," J. Community Psychol., vol. 14, no. 1, pp. 6-23, 1986.

[8] K. F. Osterman, "Students' need for belonging in the school community," Rev. Educ. Res., vol. 70, no. 3, pp. 323-367, 2000.

[9] K. K., "A sense of belonging in a university community: A study of undergraduate students," Int. J. Psychol. Behav. Sci., vol. 4, no. 1, pp. 16-20, 2014.

[10] S. Cheryan, "Strategies of Belonging," no. June, 2007.

[11] S. T. Fiske and S. E. Taylor, Social cognition. Mcgraw-Hill Book Company, 1991.

[12] P. A. Schutz, R. Pekrun, and G. D. Phye, Emotion in education, vol. 10. Elsevier, 2007.

[13] N. N. Kellam, T. Costantino, J. Walther, and N. W. Sochacka, "Uncovering the role of emotion in engineering education within an integrated curricular experience," in American Society for Engineering Education, 2011.

[14] W. Zorman and H. M. College, "But how do you feel?," in American Society for Engineering Education, 2018.

[15] S. Lyubomirsky, L. King, and E. Diener, "The benefits of frequent positive affect: does happiness lead to success?," Psychol. Bull., vol. 131, no. 6, pp. 803-855, 2005.

[16] A. L. Reschly, E. S. Huebner, J. J. Appleton, and S. Antaramian, "Engagement as flourishing: The contribution of positive emotions and coping to adolescents' engagement at school and with learning," Psychol. Sch., vol. 45, no. 5, pp. 419-431, 2008.

[17] J. C. Turner, P. K. Thorpe, and D. K. Meyer, "Students' reports of motivation and negative affect: A theoretical and empirical analysis," J. Educ. Psychol., vol. 90, no. 4, pp. 758-771, 1998.

[18] M. Moon, H. Jeon, and S. Kwon, "Effect of gender on students' emotion with genderrelated public self-consciousness as a moderator in mixed-gender physical education classes," Sch. Psychol. Int., vol. 37, no. 5, pp. 470-484, 2016.

[19] L. J. Sax, A. N. Bryant, and S. K. Gimartin, "A longitudinal investigation of emotional health among first-year college students: Comparisons of women and men," in Annual Meeting of the Association for the Study of Higher Education, 2002.

[20] J. Husman, K. C. Cheng, K. Puruhito, and E. J. Fishman, "Understanding engineering students stress and emotions during an introductory engineering course," in American Society for Engineering Education, 2015.

[21] A. Godwin, G. Potvin, Z. Hazari, and R. Lock, "Identity, critical agency and engineering: An affective model for predicting engineering as a career choice," J. Eng. Educ., vol. 105, no. 2, pp. 312-340, 2016.

[22] K. Luchini-Colbry, K. S. Wawryzynski, and M. Shannahan, "Feeling like a grad student: a survey of undergraduate researchers' expectations and experiences," in American Society for Engineering Education, 2013.

[23] S. Hareli and U. Hess, "A cross-cultural study on emotion expression and the learning of social norms," vol. 6, no. October, pp. 1-12, 2015.

[24] N. Lim, "Cultural differences in emotion: differences in emotional arousal level between the East and the West," Integr. Med. Res., vol. 5, no. 2, pp. 105-109, 2016.

[25] J. A. Russell, “A circumplex model of affect.," J. Pers. Soc. Psychol., vol. 39, no. 6, p. $1161,1980$.

[26] L. A. Feldman, "Distinguishing depression and anxiety in self-report: Evidence from 
confirmatory factor analysis on nonclinical and clinical samples.," J. Consult. Clin. Psychol., vol. 61, no. 4, p. 631, 1993.

[27] J. L. Tsai, "Ideal affect: Cultural causes and behavioral consequences," Perspect. Psychol. Sci., vol. 2, no. 3, pp. 242-259, 2007.

[28] E. K. Suh and J. S. Koo, "A concise measure of subjective well-being (COMOSWB): Scale development and validation," Korean J. Soc. Personal. Psychol., vol. 25, no. 1, pp. 95-113, 2011.

[29] E. A. Plant, J. S. Hyde, D. Keltner, and P. G. Devine, "The Gender Stereotyping of Emotions," Psychol. Women Q., vol. 24, pp. 81-92, 2000.

[30] J. H. Pleck, F. L. Sonenstein, and L. C. Ku, "Masculinity ideology and its correlates," Gend. Psychol. Read., vol. 49, no. 3, pp. 308-326, 1993.

[31] B. Markway, "How to crack the code of men's feelings," Psychology Today, 2014. .

[32] A. J. Franklin and N. Boyd-Franklin, "Invisibility syndrome: A clinical model of the effects of racism on African-American males," Am. J. Orthopsychiatry, vol. 70, no. 1, pp. 33-41, 2000.

[33] J. C. Wade, "Masculinity ideology, male reference group identity dependence, and African American men's health-related attitudes and behaviors," Psychol. Men Masc., vol. 9, no. 1, pp. 5-16, 2008.

[34] W. H. Courtenay, "Constructions of masculinity and their influence on men's well-being: a theory of gender and health," Soc. Sci. Med., vol. 50, no. 10, pp. 1385-1401, 2000.

[35] L. Hirshfield, J. Whinnery, D. M. Gilbuena, and M. D. Koretsky, "A study of feedback provided to student teams engaged in open-ended projects," in American Society for Engineering Education, 2014.

[36] L. Hirshfield, M. Dailey, and S. Edington, "Work in Progress: Common Reading Experience: Assessing the impact on perceptions, identity, and belonging among first-year engineering students," in American Society for Engineering Education, 2018.

[37] J. W. Creswell, Research Design: Qualitative, Quantitative and Mixed-Methods Approaches, 3rd ed. Thousand Oaks, CA: SAGE Publications, Inc., 2009. 